\title{
Discrete symmetries, invisible axion, and lepton number symmetry in an economic 3-3-1 model
}

\author{
Alex G. Dias* \\ Instituto de Física, Universidade de São Paulo, Caixa Postal 66.318, 05315-970, São Paulo-SP, Brazil \\ C. A de S. Pires ${ }^{\dagger}$ \\ Departamento de Física, Universidade Federal da Paraíba, Caixa Postal 5008, 58051-970, João Pessoa-PB, Brazil \\ P. S. Rodrigues da Silva \\ Instituto de Física Teórica, Universidade Estadual Paulista, Rua Pamplona 145, 01405-900 São Paulo-SP, Brazil
}

(Received 12 September 2003; published 30 December 2003)

\begin{abstract}
We show that Peccei-Quinn and lepton number symmetries can be a natural outcome in a 3-3-1 model with right-handed neutrinos after imposing a $Z_{11} \otimes Z_{2}$ symmetry. This symmetry is suitably accommodated in this model when we augment its spectrum by including merely one singlet scalar field. We work out the breaking of the Peccei-Quinn symmetry, yielding the axion, and study the phenomenological consequences. The main result of this work is that the solution to the strong $C P$ problem can be implemented in a natural way, implying an invisible axion phenomenologically unconstrained, free of domain wall formation, and constituting a good candidate for the cold dark matter.
\end{abstract}

DOI: 10.1103/PhysRevD.68.115009

PACS number(s): $12.60 . \mathrm{Cn}, 14.80 . \mathrm{Mz}$

\section{INTRODUCTION}

The standard model (SM) of strong and electroweak interactions $S U_{C}(3) \otimes S U_{L}(2) \otimes U_{Y}(1)$ has shown its extraordinary accuracy in explaining many features of particle physics along the years. Among the issues not covered by this successful model there is the fact that the QCD vacuum has a nontrivial structure revealed by its nonperturbative regime, implying the so-called strong- $C P$ or $\theta$ problem (the subject is widely reviewed in Ref. [1]). Violation of $C P$ by strong interactions appears in the theory after the introduction of an instanton solution to solve the $U_{A}(1)$ problem [2]. It induces the so-called $\theta$ term in the QCD Lagrangian, which violates $P, T$, and $C P$. Additional electroweak effects change this term proportionally to $\operatorname{Det}[M]$, where $M$ is the quark mass matrix. The effective $\theta$ term $\theta_{\text {eff }}$ is observable through the electric dipole moment of the neutron, whose experimental bound implies the upper limit $\left|\theta_{\text {eff }}\right|<10^{-9}$ [3]. The smallness of $\theta_{\text {eff }}$ is what we call the strong- $C P$ problem.

Among the several solutions proposed to solve the strong- $C P$ problem, there is one which is particularly elegant. It was introduced by Peccei and Quinn [4] and consists of imposing a global chiral symmetry, known as the Peccei-Quinn (PQ) symmetry $U_{P Q}(1)$, on the classical Lagrangian so that the dynamics of the theory sets $\theta_{\text {eff }}$ to zero. Because of the breaking of PQ symmetry, a massless pseudoscalar is generated, the axion, which couples linearly to the axial anomaly. When this axion develops a vacuum expectation value $(\mathrm{VEV}) v_{P Q}$, it produces a further displacement on $\theta_{\text {eff }}$, making it disappear in favor of a dynamical field, the physical axion, eliminating the strong- $C P$ violating term of the theory. The breaking of PQ symmetry brings a

\footnotetext{
*Electronic address: alexdias@fma.if.usp.br

†Electronic address: cpires@ fisica.ufpb.br

‡Electronic address: fedel@ift.unesp.br
}

new scale into the theory, $f_{P Q}$, the axion decay constant, bounded by astrophysical and cosmological data, and its allowed range is $10^{9} \mathrm{GeV}<f_{P Q}<10^{12} \mathrm{GeV}$ [5].

The first class of models introducing the axion via PQ symmetry in the context of the SM was obtained by Weinberg and Wilczek [6]. This axion was soon shown to be unrealistic mainly due to its nonsuppressed coupling to light matter fields [7], which happens when $v_{P Q}$ is of order of the electroweak scale. Viable models to solve the strong $C P$, introducing an invisible axion, were devised by Kim and independently by Shifman, Vainshtein, and Zakharov [8] (KSVZ) and by Dine, Fischler, and Srednicki as well as Zhitnitskii [9] (DFSZ). Both make axions invisible by increasing $v_{P Q}$ (the larger $v_{P Q}$, the weaker the axion-matter coupling) and obtain the axion through a singlet scalar. In the KSVZ axion model the ordinary quarks and leptons do not carry PQ charges; some heavy new quarks have to be included which carry this quantum number. On the contrary, in the DFSZ model ordinary quarks and leptons do carry PQ charges, although these fermions do not couple directly to the singlet, which happens only at the loop level through interactions in the potential.

The possibility of an invisible axion makes the PQ approach even more attractive since in this case the axion is a natural candidate for explaining the existence of cold dark matter (CDM) [10]. This is possible because the axion receives a tiny mass through a chiral anomaly, $m_{a}^{2}$ $\sim \Lambda_{Q C D}^{4} / f_{P Q}^{2}$, amounting to a mass of $\mathcal{O}\left(10^{-5}\right) \mathrm{eV}$. However, it is not easy, in general, to obtain the required PQ symmetry in a natural way; most models have to impose it from the beginning, weakening such a solution to the strong- $C P$ problem. That is the reason we concentrate here on a class of models where the symmetry would arise automatically: namely, a particular version of the $S U_{C}(3)$ $\otimes S U_{L}(3) \otimes U_{Y}(1)$ model (3-3-1 for short) [11-15].

In 3-3-1 models the anomaly cancellation requires a minimal of three families (or a multiple of three in larger ver- 
sions). Besides, there is a bunch of new particles and interactions which make these models phenomenologically rich and attractive as an alternative to the SM. If we assume that in the realm of intermediate energy there are no exotic leptons, then the 3-3-1 symmetry allows for only two possible gauge models for the strong and electroweak interactions, which will be referred as version I and version II.

In the most popular one, version I [11], the triplet of leptons is composed of $\left(\nu_{L}, l_{L}, l_{R}^{c}\right)^{T}$; it contains exotic quarks with electric charge $4 / 3$ and $5 / 3$ and a doubly charged bilepton gauge boson $U^{ \pm \pm}$, which prompts rare lepton decays. It also implies an upper bound on the Weinberg angle, $\sin \left(\theta_{W}\right)<1 / 4$. Version II is the 3-3-1 model with right-handed neutrinos [12]. In it the triplet of leptons is constituted by $\left(\nu_{L}, l_{L}, \nu_{R}^{c}\right)^{T}$. Its bilepton gauge boson is neutral and their exotic quarks carry the usual charges $1 / 3$ and $2 / 3$ [16].

The physical properties of these models were investigated in several works and their different aspects became evident $[14,15]$. Among these differences it is noticeable that version I requires a minimal of three triplets and one sextet of scalars in order to generate the masses for all fermions and gauge bosons while version II does the same job with only three triplets.

Their shared aspects include the naturalness of massive neutrinos, with the difference that in version I neutrinos are Majorana type, while in version II they are Dirac type. Besides, from their structure these models dispose of enough constraints upon the $U(1)_{N}$ quantum numbers, leading to a correct pattern for electric charge quantization [17]. Another of these aspects is that also the PQ symmetry and the leptonic symmetry can emerge naturally in both versions [1820].

Since version II of 3-3-1 was observed to possess the PQ symmetry with a smaller content [20], although in that context the axion was of the Weinberg-Wilczek kind, we decided to chose this more economical model and investigate the possibility of obtaining an invisible axion by including only one extra scalar singlet field in the model. The presence of CDM candidates in version II of 3-3-1 was recently addressed [21], but here we wish to have the axion playing such a role. There is a crucial issue that has to be addressed when trying to stick with a CDM singlet axion though. It concerns the fact that gravitation induces dangerous effective terms in the Lagrangian, explicitly breaking any global symmetry of the theory. In particular, focusing on $U_{P Q}(1)$, this breaking implies a huge contribution to the axion mass. There remains the question of whether an appropriate mechanism exists in order to avoid such terms, stabilizing the axion. Fortunately, the annoying terms can be conveniently suppressed by the presence of suitable discrete symmetries. Moreover, it was noticed in the late 1980s by Kraus and Wilczek [22] that a local continuous symmetry at high energies manifests at low energies as discrete (local) symmetries which, differently from global ones, are expected to be respected by gravity. This means that the needed discrete symmetries can arise in a rather natural way if we assume some underlying local continuous symmetry.

Discrete gauge symmetries have been used to stabilize the axion in a model with extra dimensions by Kamionkowsky and March-Russell [23] more than ten years ago. It was also pointed out that large discrete symmetries can naturally arise in the context of string theories [24]. The same is true in dimensional deconstruction [25], leading to low-mass pseudo-Nambu-Goldstone bosons as axion and quintessence. Also, in an attempt to prevent $B-L$ violation in a class of supersymmetric standard model, large discrete symmetries were imposed, implying an automatic PQ symmetry, stabilized against quantum gravity effects [26]. For what we are concerned with, it was noticed in Ref. [19] that 3-3-1 models possess a large enough number of fields to accommodate large discrete symmetries $Z_{N}$. And the larger $N$ is, the higher are the number of suppressed unwanted terms in the Lagrangian. In order to obtain a $Z_{13}$ symmetry, the authors in Ref. [19] added some extra fermion fields to the model, resulting in an automatic PQ symmetry and the axion protected under gravitational mass corrections. This constitutes an additional motivation for considering these 3-3-1 models to obtain the invisible axion and solve the strong- $C P$ problem.

This work is divided as follows. We first introduce the model in Sec. II. In Sec. III we impose a $Z_{11} \otimes Z_{2}$ symmetry, associating the appropriate charges for the fields, and obtain that the resulting Lagrangian is invariant under $U_{P Q}(1)$, identifying the correct PQ charges. This is done within the same spirit as that presented in Refs. [19,27], assigning charges under a discrete symmetry group to the fields at hand and observing that a PQ symmetry emerges automatically if a $Z_{2}$ is also imposed. We will see that in this case also lepton number symmetry arises naturally. In Sec. IV, we analyze the symmetry breaking pattern of the model, recognizing the axion and its couplings. We finally present the conclusions in Sec. V.

\section{MODEL}

Our investigation on this work relies on version II of the 3-3-1 models [15]. Its lepton content comes in the fundamental representation of the $S U(3)_{L}$, the left-handed composing a triplet and the right-handed a singlet,

$$
f_{L}^{a}=\left(\begin{array}{c}
\nu_{L}^{a} \\
e_{L}^{a} \\
\left(\nu_{R}^{c}\right)^{a}
\end{array}\right) \sim(1,3,-1 / 3), \quad e_{a R} \sim(1,1,-1),
$$

with $a=1,2,3$ representing the three known generations. We are indicating the transformation under 3-3-1 after the similarity sign " ." Differently from version I, right-handed neutrinos are already present instead of exotic leptons.

In the quark sector, one generation of left-handed fields comes in the triplet fundamental representation of $S U(3)_{L}$ and the other two compose an antitriplet with the content

$$
Q_{i L}=\left(\begin{array}{c}
d_{i L} \\
-u_{i L} \\
d_{i L}^{\prime}
\end{array}\right), \quad Q_{3 L}=\left(\begin{array}{c}
u_{3 L} \\
d_{3 L} \\
u_{3 L}^{\prime}
\end{array}\right)
$$

transforming as $(3, \overline{3}, 0)$ and $(3,3,1 / 3)$, respectively, and $i$ $=1,2$ represents two different generations. The primed 
quarks are the exotic ones but with the usual electric charges. Right-handed quark fields are singlets:

$$
\begin{aligned}
& u_{i R} \sim(3,1,2 / 3), \quad d_{i R} \sim(3,1,-1 / 3), \quad d_{i R}^{\prime} \sim(3,1,-1 / 3) \\
& u_{3 R} \sim(3,1,2 / 3), \quad d_{3 R} \sim(3,1,-1 / 3), \quad u_{3 R}^{\prime} \sim(3,1,2 / 3) .
\end{aligned}
$$

In order to generate the masses for the gauge bosons and fermions, the model requires only three triplets of scalars: namely,

$$
\chi=\left(\begin{array}{c}
\chi^{0} \\
\chi^{-} \\
\chi^{\prime 0}
\end{array}\right), \quad \eta=\left(\begin{array}{c}
\eta^{0} \\
\eta^{-} \\
\eta^{\prime 0}
\end{array}\right), \quad \rho=\left(\begin{array}{c}
\rho^{+} \\
\rho^{0} \\
\rho^{\prime+}
\end{array}\right),
$$

where $\eta$ and $\chi$ both transform as $(1,3,-1 / 3)$ and $\rho$ transforms as $(1,3,2 / 3)$.

With these scalars and matter fields we can write the Yukawa interactions [28]

$$
\begin{aligned}
\mathcal{L}^{Y}= & G_{1} \bar{Q}_{3 L} u_{3 R}^{\prime} \chi+G_{2}^{i j} \bar{Q}_{i L} d_{j R}^{\prime} \chi^{*}+G_{3}^{3 a} \bar{Q}_{3 L} u_{a R} \eta \\
& +G_{4}^{i a} \bar{Q}_{i L} d_{a R} \eta^{*}+G_{5}^{3 a} \bar{Q}_{3 L} d_{a R} \rho+G_{6}^{i a} \bar{Q}_{i L} u_{a R} \rho^{*} \\
& +h_{a b} \bar{f}_{a L} e_{b R} \rho+h_{a b}^{\prime} \epsilon^{i j k}\left(\bar{f}_{a L}\right)_{i}\left(f_{b L}\right)_{j}^{c}\left(\rho^{*}\right)_{k}+\text { H.c. }
\end{aligned}
$$

After the breaking of the 3-3-1 symmetry the vector gauge bosons $W^{ \pm}, V^{ \pm}, U^{0}$, and $U^{0 \dagger}$ interact with matter as follows [29]:

$$
\begin{aligned}
\mathcal{L}^{C I}= & -\frac{g}{\sqrt{2}}\left[\bar{\nu}_{L}^{a} \gamma^{\mu} e_{L}^{a} W_{\mu}^{+}+\left(\bar{\nu}_{R}^{c}\right)^{a} \gamma^{\mu} e_{L}^{a} V_{\mu}^{+}+\bar{\nu}_{L}^{a} \gamma^{\mu}\left(\nu_{R}^{c}\right)^{a} U_{\mu}^{0}\right. \\
& +\bar{u}_{L}^{a} \gamma^{\mu} d_{L}^{a} W_{\mu}^{+}+\left(\bar{u}_{3 L}^{\prime} \gamma^{\mu} d_{3 L}+\bar{u}_{i L} \gamma^{\mu} d_{i L}^{\prime}\right) V_{\mu}^{+} \\
& \left.+\left(\bar{u}_{3 L} \gamma^{\mu} u_{3 L}^{\prime}-\bar{d}_{i L}^{\prime} \gamma^{\mu} d_{i L}\right) U_{\mu}^{0}\right]+ \text { H.c. }
\end{aligned}
$$

It is through these Lagrangian interactions $\mathcal{L}^{Y}$ and $\mathcal{L}^{C I}$ that we can recognize particles that carry lepton number $L$ such that total lepton number is conserved at this level. From these interactions we have

$$
L\left(V^{+}, u_{3}^{\prime}, \eta^{\prime 0}, \rho^{++}\right)=-2, \quad L\left(U^{0}, d_{i}^{\prime}, \chi^{0}, \chi^{-}\right)=+2 .
$$

Notice that the new quarks $u_{3}^{\prime}$ and $d_{i}^{\prime}$ are leptoquarks once they carry lepton and baryon numbers; $V^{ \pm}$are charged vector bileptons while $U^{0}$ and $U^{0 \dagger}$ are neutral vector bileptons. We have also charged scalar bileptons and two neutral scalar bileptons. These last ones would be important in studying spontaneous breaking of lepton number if the associated global symmetry is conserved by the potential, leading to the so-called majoron, as discussed in Ref. [20].

We include also an additional singlet scalar field $\phi$ $\sim(1,1,0)$ in order to complete the spectrum, allowing for the desired discrete symmetry which will enable us to get an axion protected under a large gravitational contribution to its mass.

Finally, we can write the most general, renormalizable, and gauge-invariant potential for this model. We divide into two pieces: one Hermitian, $V_{H}$, and one non-Hermitian, $V_{N H}$, which can be written as

$$
\begin{aligned}
V_{H}= & \mu_{\phi}^{2} \phi^{2}+\mu_{\chi}^{2} \chi^{2}+\mu_{\eta}^{2} \eta^{2}+\mu_{\rho}^{2} \rho^{2}+\lambda_{1} \chi^{4}+\lambda_{2} \eta^{4}+\lambda_{3} \rho^{4}+\lambda_{4}\left(\chi^{\dagger} \chi\right)\left(\eta^{\dagger} \eta\right)+\lambda_{5}\left(\chi^{\dagger} \chi\right)\left(\rho^{\dagger} \rho\right)+\lambda_{6}\left(\eta^{\dagger} \eta\right)\left(\rho^{\dagger} \rho\right)+\lambda_{7}\left(\chi^{\dagger} \eta\right) \\
& \times\left(\eta^{\dagger} \chi\right)+\lambda_{8}\left(\chi^{\dagger} \rho\right)\left(\rho^{\dagger} \chi\right)+\lambda_{9}\left(\eta^{\dagger} \rho\right)\left(\rho^{\dagger} \eta\right)+\lambda_{10}\left(\phi \phi^{*}\right)^{2}+\lambda_{11}\left(\phi \phi^{*}\right)\left(\chi^{\dagger} \chi\right)+\lambda_{12}\left(\phi \phi^{*}\right)\left(\rho^{\dagger} \rho\right)+\lambda_{13}\left(\phi \phi^{*}\right)\left(\eta^{\dagger} \eta\right)
\end{aligned}
$$

and

$$
\begin{aligned}
V_{N H}= & \mu_{\chi \eta}^{2} \chi^{\dagger} \eta+f_{1} \chi^{\dagger} \eta \phi+f_{2} \chi^{\dagger} \eta \phi^{*}+\lambda_{14}\left(\chi^{\dagger} \eta\right)^{2}+\lambda_{15} \chi^{\dagger} \eta \phi \phi+\lambda_{16} \chi^{\dagger} \eta \phi^{*} \phi+\lambda_{17} \chi^{\dagger} \eta \phi^{*} \phi^{*}+\frac{1}{\sqrt{2}} \epsilon^{i j k}\left(f_{3} \eta_{i} \rho_{j} \chi_{k}\right. \\
& \left.+f_{4} \eta_{i} \eta_{j} \rho_{k}+f_{5} \chi_{i} \chi_{j} \rho_{k}\right)+\epsilon^{i j k}\left(\lambda_{18} \eta_{i} \rho_{j} \chi_{k}+\lambda_{19} \eta_{i} \eta_{j} \rho_{k}+\lambda_{20} \chi_{i} \chi_{j} \rho_{k}\right) \phi+\epsilon^{i j k}\left(\lambda_{21} \eta_{i} \rho_{j} \chi_{k}+\lambda_{22} \eta_{i} \eta_{j} \rho_{k}+\lambda_{23} \chi_{i} \chi_{j} \rho_{k}\right) \phi^{*} \\
& +\lambda_{24}\left(\chi^{\dagger} \rho\right)\left(\rho^{\dagger} \eta\right)+\lambda_{25}\left(\chi^{\dagger} \eta\right)\left(\eta^{\dagger} \eta\right)+\lambda_{26}\left(\chi^{\dagger} \eta\right)\left(\rho^{\dagger} \rho\right)+\lambda_{27}\left(\chi^{\dagger} \eta\right)\left(\chi^{\dagger} \chi\right)+\text { H.c. }
\end{aligned}
$$

With this at hand we have all the necessary ingredients to associate a discrete symmetry $Z_{11}$ to the model. This will allow us to eliminate several terms in the non-Hermitian potential, Eq. (9), and verify that we need only an additional $Z_{2}$ symmetry to have PQ symmetry naturally, assigning the appropriate PQ charges to fermions and scalars.

\section{III. $Z_{11}$ AND PQ SYMMETRIES}

A discrete symmetry $Z_{N}$ can naturally be accommodated when the theory has a large enough number of fields in its spectrum. It was observed that this is the case for the SM when some scalar multiplets and right-handed neutrinos are 
added [27] or for the minimal 3-3-1 model when only righthanded neutrinos need to be included [19]. It was obtained that a $Z_{13}$ local symmetry could be imposed in this way, leading to a natural PQ symmetry. We remark that this idea was first pursued by Lazarides et al. in the context of models embedded in superstring theories [30].

Here we are going to apply such idea to the version of 3-3-1 model presented in Sec. II, which has right-handed neutrinos in its fundamental representation. It was observed that an axion might be a natural outcome when a $Z_{2}$ symmetry was imposed in this model [19]. Although this axion is of the Weinberg-Wilczek kind, thus phenomenologically discarded [7], if we consider the enlarged spectrum with a singlet scalar $\phi$, the axion can be a mixing of this field with other scalars in the model, with its major component being the pseudoscalar part of the $\phi$ field. Then, a discrete symmetry can be imposed, allowing for an axion also protected under gravitational mass corrections.

To proceed in this way we first assign the $Z_{N}$ charges to all independent fields and check for additional symmetries appearing after eliminating forbidden terms under $Z_{N}$. It will turn out that a chiral $U(1)$ symmetry arise, and we will see that it is possible to identify it with PQ symmetry. It would be interesting to have a $Z_{13}$ symmetry so as to obtain a PQ scale in its upper limit $v_{P Q} \sim 10^{12} \mathrm{GeV}$. Although the model disposes of 14 independent multiplets, it is not possible to accommodate a symmetry greater than $Z_{12}$, because the Yukawa interactions in Eq. (5) imply some constraints over the allowed $Z_{N}$ charges. It is clear that $N=12$ is the value of $N$ that allows for a maximal protection of the axion under gravitational effects in this model. However, besides the seemingly difficulty of avoiding to repeat the phases of the multiplets, the singlet $\phi$ would have to acquire a very specific phase since 12 is not a prime number. In other words, any even phase would make the transformation to belong to a smaller discrete symmetry, jeopardizing our intent of suppressing some high-order operators involving $\phi$ products. For this reason the largest discrete symmetry we can use is $Z_{11}$, which allows any phase to $\phi$, except the trivial one.

The effective operators responsible for the gravitational mass contribution are of the form $\phi^{n} / M_{P l}^{n-4}$. A $Z_{N}$ symmetry automatically suppress terms of this kind until some $n=N$ -1 . The main surviving term contributing to the axion mass is the one with $n=N$. It is true that with $Z_{11}$ the axion is protected only for energy scales not bigger than $\langle\phi\rangle$ $\simeq 10^{10} \mathrm{GeV}$. Nevertheless, this is not a threat for the model since we still have values for the $\theta$ angle and axion mass (gravitationally induced) [31]:

$$
\begin{aligned}
M_{a}^{g r a v} & \simeq \sqrt{\frac{\langle\phi\rangle^{N-2}}{M_{P l}^{N-4}}} \simeq 10^{-12} \mathrm{eV} \simeq 10^{-7} m_{a}, \\
\theta_{e f f} & \simeq \frac{\langle\phi\rangle^{N}}{M_{P l}^{N-4} \Lambda_{Q C D}^{4}} \simeq 10^{-19},
\end{aligned}
$$

where we have used $M_{P l} \simeq 10^{19} \mathrm{GeV}$ and $\Lambda_{Q C D}$ $\simeq 300 \mathrm{MeV}$, and $m_{a} \simeq 10^{-5} \mathrm{eV}$ is the instanton induced axion mass. These values are consistent with astrophysical and experimental bounds (see PDG [3]). If we had taken $\langle\phi\rangle$ $\simeq 10^{11} \mathrm{GeV}$, the axion would still be protected under gravitation, but the $\theta$ value would be on the threshold of its bound $\theta_{\text {eff }} \leqslant 10^{-9}$. So we can have a valid solution to the strong- $C P$ problem for $Z_{11}$ for scales $\langle\phi\rangle \lesssim 10^{10} \mathrm{GeV}$ in this version of 3-3-1. In order to seek for this solution let us proceed further by first assigning the correct $Z_{11}$ charges to the fields. Defining $\omega_{k} \equiv e^{2 \pi i k / 11}\{k=0, \pm 1, \ldots, \pm 5\}$, the $Z_{11}$ transformations are given by

$$
\begin{aligned}
\phi & \rightarrow \omega_{1} \phi, \quad f_{a L} \rightarrow \omega_{1}^{-1} f_{a L}, \\
\rho & \rightarrow \omega_{2} \rho, \quad d_{a R} \rightarrow \omega_{2}^{-1} d_{a R}, \\
\chi & \rightarrow \omega_{3} \chi, \quad\left(e_{R}, u_{3 R}^{\prime}\right) \rightarrow \omega_{3}^{-1}\left(e_{R}, u_{3 R}^{\prime}\right), \\
Q_{i L} & \rightarrow \omega_{4} Q_{i L}, \quad d_{i R}^{\prime} \rightarrow \omega_{4}^{-1} d_{i R}^{\prime}, \\
\eta & \rightarrow \omega_{5} \eta, \quad u_{a R} \rightarrow \omega_{5}^{-1} u_{a R}, \\
Q_{3 L} & \rightarrow \omega_{0} Q_{3 L} .
\end{aligned}
$$

At this point it is possible to go back to the potential, Eq. (9), and note that this symmetry eliminates all non-Hermitian terms except three: namely, $\chi^{\dagger} \eta \phi^{*} \phi^{*}, \eta \rho \chi \phi, \eta \eta \rho \phi^{*}$.

If besides the $Z_{11}$ symmetry we impose a $Z_{2}$ symmetry that acts as

$$
\left(\phi, \chi, d_{R}^{\prime}, u_{3 R}^{\prime}\right) \rightarrow-\left(\phi, \chi, d_{R}^{\prime}, u_{3 R}^{\prime}\right),
$$

with the remaining fields transforming trivially, the only term which remains in the non-Hermitian potential is the $\eta \rho \chi \phi$. It should be noted that the Yukawa interactions in Eq. (5) do not allow for terms which interchange $\chi \leftrightarrow \eta$, since they do not respect $Z_{11} \otimes Z_{2}$ given by Eqs. (11) and (12).

We have the stage settled to see that an automatic PQ symmetry arises in the model. To achieve this conclusion we start by assigning the PQ quantum numbers such that quarks of opposite chiralities have opposite charges, yielding chiral quarks under a $U_{P Q}(1)$ transformation:

$$
\begin{aligned}
& u_{a L} \rightarrow e^{-i \alpha X_{u}} u_{a L}, \quad u_{a R} \rightarrow e^{i \alpha X_{u}} u_{a R}, \\
& u_{3 L}^{\prime} \rightarrow e^{-i \alpha X_{u}^{\prime}} u_{3 L}^{\prime}, \quad u_{3 R}^{\prime} \rightarrow e^{i \alpha X_{u}^{\prime}} u_{3 R}^{\prime}, \\
& d_{a L} \rightarrow e^{-i \alpha X_{d}} d_{a L}, \quad d_{a R} \rightarrow e^{i \alpha X_{d}} d_{a R}, \\
& d_{i L}^{\prime} \rightarrow e^{-i \alpha X_{d}^{\prime}} d_{i L}^{\prime}, \quad d_{i R}^{\prime} \rightarrow e^{i \alpha X_{d}^{\prime}} d_{i R}^{\prime} .
\end{aligned}
$$

For the leptons we can define their PQ charges by

$$
\begin{aligned}
& e_{a L} \rightarrow e^{i \alpha X_{e}} e_{a L}, \quad e_{a R} \rightarrow e^{i \alpha X_{e R}} e_{a R}, \\
& \nu_{a L} \rightarrow e^{i \alpha X_{\nu}} \nu_{a L}, \quad \nu_{a R} \rightarrow e^{i \alpha X_{\nu R}} \nu_{a R} .
\end{aligned}
$$

With these assignments and taking the Yukawa interactions in Eq. (5) into account, as well as the non-Hermitian terms $\eta \rho \chi \phi$, we easily see that the PQ charges for the scalars are constrained and imply the relations 


$$
X_{d}=-X_{u}, \quad X_{d^{\prime}}=-X_{u^{\prime}}, X_{\nu}=X_{e R}, X_{e}=X_{\nu R} .
$$

We can make the further choice $X_{d}=X_{d^{\prime}}$, leading to

$$
X_{d}=X_{d^{\prime}}=-X_{u}=-X_{u^{\prime}}=-X_{e}=X_{e R}=X_{\nu}=-X_{\nu R},
$$

implying that the PQ symmetry is chiral for the leptons too, and the scalars transform as

$$
\begin{gathered}
\phi \rightarrow e^{-2 i \alpha X_{d}} \phi, \quad \eta^{0} \rightarrow e^{2 i \alpha X_{d}} \eta^{0}, \\
\eta^{-} \rightarrow \eta^{-}, \quad \eta^{\prime 0} \rightarrow e^{2 i \alpha X_{d}} \eta^{\prime 0}, \\
\rho^{+} \rightarrow \rho^{+}, \quad \rho^{0} \rightarrow e^{-2 i \alpha X_{d}} \rho^{0}, \\
\rho^{++} \rightarrow \rho^{++}, \quad \chi^{0} \rightarrow e^{2 i \alpha X_{d}} \chi^{0}, \\
\chi^{-} \rightarrow \chi^{-}, \quad \chi^{\prime 0} \rightarrow e^{2 i \alpha X_{d}} \chi^{\prime 0} .
\end{gathered}
$$

Now it is transparent that the whole Lagrangian of the model is $U_{P Q}(1)$ invariant and the strong- $C P$ problem can be solved in the context of this model. The strong- $C P$ violation angle is given by the sum over the quarks PQ charges, which translates to

$$
\theta \rightarrow \theta \pm 2 \alpha X_{d}
$$

This result is possible in this version of 3-3-1 because the PQ charges of the exotic quarks $d^{\prime}$ and $u_{3}^{\prime}$ do not cancel exactly for the case of interest here, $X_{u}=-X_{d}$. Moreover, the model is particularly attractive in the sense it does not present the domain wall problem [32]. This means that there is no discrete subset of PQ symmetry that leaves the axion potential invariant-i.e., $\nexists Z_{N} \subset U_{P Q}(1)$ such that $V_{\text {axion }}(\theta)$ is invariant. This is similar to what happens in the 3-3-1 version discussed in Ref. [19], although there right-handed neutrinos had to be added to the model besides the singlet scalar.

It is remarkable that under $Z_{11} \otimes Z_{2}$, not only is the PQ chiral symmetry automatic but the lepton number symmetry also appears naturally in the model, once the possibly nonconserving lepton number terms present in the potential completely disappeared. In this sense, discrete symmetries originating at some high-energy scale seem to be enough to generate the desired global symmetries we need at lower energies.

We finally write the most general potential invariant under 3-3-1 and $Z_{11} \otimes Z_{2}$ [or $U_{P Q}(1)$ and lepton number] symmetries,

$$
V(\eta, \rho, \chi)=V_{H}+\lambda_{\phi} \epsilon^{i j k} \eta_{i} \rho_{j} \chi_{k} \phi+\text { H.c., }
$$

where $V_{H}$ is given in Eq. (8).

In the next section we are going to use this potential to recognize the axion, the Goldstone boson originating from the breaking of the PQ symmetry, and verify that it is constituted mostly of the singlet $\phi$.

\section{SPONTANEOUSLY BROKEN PQ SYMMETRY}

The potential given in the previous section, Eq. (19), allows us to obtain the mass eigenstates for the scalars, so we can identify the Goldstone bosons which are absorbed by the massive gauge bosons and extract the axion in terms of the interaction eigenstates. To accomplish this, let us consider that only $\chi^{\prime 0}, \rho^{0}, \eta^{0}$ and $\phi$ develop a VEV and expand such fields around their VEV's in the standard way,

$$
\begin{gathered}
\chi^{\prime 0}=\frac{1}{\sqrt{2}}\left(v_{\chi^{\prime}}+R_{\chi^{\prime}}+i I_{\chi^{\prime}}\right), \quad \eta^{0}=\frac{1}{\sqrt{2}}\left(v_{\eta}+R_{\eta}+i I_{\eta}\right), \\
\rho^{0}=\frac{1}{\sqrt{2}}\left(v_{\rho}+R_{\rho}+i I_{\rho}\right), \quad \phi=\frac{1}{\sqrt{2}}\left(v_{\phi}+R_{\phi}+i I_{\phi}\right) .
\end{gathered}
$$

With such an expansion, the next step is to get the constraints that lead to the minimum of the potential:

$$
\begin{gathered}
\mu_{\chi}^{2}+\lambda_{1} v_{\chi^{\prime}}^{2}+\frac{\lambda_{4}}{2} v_{\eta}^{2}+\frac{\lambda_{5}}{2} v_{\rho}^{2}+\frac{\lambda_{11}}{2} v_{\phi}^{2}+\frac{A}{v_{\chi^{\prime}}^{2}}=0, \\
\mu_{\eta}^{2}+\lambda_{2} v_{\eta}^{2}+\frac{\lambda_{4}}{2} v_{\chi^{\prime}}^{2}+\frac{\lambda_{6}}{2} v_{\rho}^{2}+\frac{\lambda_{13}}{2} v_{\phi}^{2}+\frac{A}{v_{\eta}^{2}}=0, \\
\mu_{\rho}^{2}+\lambda_{3} v_{\rho}^{2}+\frac{\lambda_{5}}{2} v_{\chi^{\prime}}^{2}+\frac{\lambda_{6}}{2} v_{\eta}^{2}+\frac{\lambda_{12}}{2} v_{\phi}^{2}+\frac{A}{v_{\rho}^{2}}=0, \\
\mu_{\phi}^{2}+\lambda_{10} v_{\phi}^{2}+\frac{\lambda_{11}}{2} v_{\chi^{\prime}}^{2}+\frac{\lambda_{12}}{2} v_{\rho}^{2}+\frac{\lambda_{13}}{2} v_{\eta}^{2}+\frac{A}{v_{\phi}^{2}}=0,
\end{gathered}
$$

where we have defined $A \equiv \lambda_{\phi} v_{\eta} v_{\rho} v_{\chi^{\prime}} v_{\phi}$. Substituting the expansion in Eq. (20) in the potential, Eq. (19), and using the constraints above, we get the mass matrix $M_{R}^{2}\left(R_{\chi}, R_{\eta^{\prime}}\right)$ for the real scalars in the basis $\left(R_{\chi}, R_{\eta^{\prime}}\right)$,

$$
\left[\begin{array}{cc}
\frac{\lambda_{7} v_{\eta}^{2}}{4}-\frac{A}{2 v_{\chi^{\prime}}^{2}} & \frac{\lambda_{7} v_{\chi^{\prime}} v_{\eta}}{4}-\frac{A}{2 v_{\chi^{\prime}} v_{\eta}} \\
\frac{\lambda_{7} v_{\chi^{\prime}} v_{\eta}}{4}-\frac{A}{2 v_{\chi^{\prime}} v_{\eta}} & \frac{\lambda_{7} v_{\chi^{\prime}}^{2}}{4}-\frac{A}{2 v_{\eta}^{2}}
\end{array}\right],
$$

and the mass matrix $M_{R}^{2}\left(R_{\chi^{\prime}}, R_{\eta}, R_{\rho}, R_{\phi}\right)$ in the basis $\left(R_{\chi^{\prime}}, R_{\eta}, R_{\rho}, R_{\phi}\right)$, 


$$
\left[\begin{array}{cc}
\lambda_{1} v_{\chi^{\prime}}^{2}-\frac{A}{2 v_{\chi^{\prime}}^{2}} & \frac{\lambda_{4} v_{\chi^{\prime}} v_{\eta}}{2}+\frac{A}{2 v_{\eta} v_{\chi^{\prime}}} \\
\ldots & \lambda_{2} v_{\eta}^{2}-\frac{A}{2 v_{\eta}^{2}} \\
\ldots & \ldots \\
\ldots & \ldots
\end{array}\right.
$$$$
\left.\begin{array}{cc}
\frac{\lambda_{5} v_{\chi^{\prime}} v_{\rho}}{2}+\frac{A}{2 v_{\rho} v_{\chi^{\prime}}} & \frac{A}{2 v_{\phi} v_{\chi^{\prime}}} \\
\frac{\lambda_{6} v_{\eta} v_{\rho}}{2}+\frac{A}{2 v_{\rho} v_{\eta}} & \frac{A}{2 v_{\eta} v_{\phi}} \\
\lambda_{3} v_{\rho}^{2}-\frac{A}{2 v_{\rho}^{2}} & \frac{A}{2 v_{\rho} v_{\phi}} \\
\ldots & \lambda_{10} v_{\phi}^{2}-\frac{A}{2 v_{\phi}^{2}}
\end{array}\right]
$$

These bases are not coupled; that is the reason we have two squared mass matrices. From the first matrix, Eq. (22), after diagonalization it is easy to recognize the following massless scalar in it:

$$
R_{G}=\frac{1}{\sqrt{v_{\eta}^{2}+v_{\chi^{\prime}}^{2}}}\left(v_{\eta} R_{\eta^{\prime}}-v_{\chi^{\prime}} R_{\chi}\right) .
$$

The other real scalar mass eigenstate is orthogonal to this one and those coming from the diagonalization of the matrix, Eq. (23), which are a little more intricate but fortunately we do not need them for our purpose.

Regarding the pseudoscalars, similarly to the real scalars, we obtain the mass matrix $M_{I}^{2}\left(I_{\chi}, I_{\eta^{\prime}}\right)$ in the basis $\left(I_{\chi}, I_{\eta^{\prime}}\right)$,

$$
\left[\begin{array}{cc}
\frac{\lambda_{7} v_{\eta}^{2}}{4}-\frac{A}{2 v_{\chi^{\prime}}^{2}} & -\frac{\lambda_{7} v_{\chi^{\prime}} v_{\eta}}{4}+\frac{A}{2 v_{\eta} v_{\chi^{\prime}}} \\
-\frac{\lambda_{7} v_{\chi^{\prime}} v_{\eta}}{4}+\frac{A}{2 v_{\eta} v_{\chi^{\prime}}} & \frac{\lambda_{7} v_{\chi^{\prime}}^{2}}{4}-\frac{A}{2 v_{\eta}^{2}}
\end{array}\right]
$$

and the mass matrix $M_{I}^{2}\left(I_{\chi^{\prime}}, I_{\eta}, I_{\rho}, I_{\phi}\right)$ in the basis $\left(I_{\chi^{\prime}}, I_{\eta}, I_{\rho}, I_{\phi}\right)$,

$$
-\frac{A}{2}\left[\begin{array}{cccc}
\frac{1}{v_{\chi^{\prime}}^{2}} & \frac{1}{v_{\eta} v_{\chi^{\prime}}} & \frac{1}{v_{\rho} v_{\chi^{\prime}}} & \frac{1}{v_{\chi^{\prime}} v_{\phi}} \\
\cdots & \frac{1}{v_{\eta}^{2}} & \frac{1}{v_{\eta} v_{\rho}} & \frac{1}{v_{\eta} v_{\phi}} \\
\ldots & \cdots & \frac{1}{v_{\rho}^{2}} & \frac{1}{v_{\eta} v_{\rho}} \\
\cdots & \cdots & \cdots & \frac{1}{v_{\phi}^{2}}
\end{array}\right] .
$$

From these matrices we can easily obtain the Goldstone bosons and identify the axion as the one whose main component is in the $\phi$ direction. The Goldstone and pseudoGoldstone bosons are

$$
\begin{aligned}
& a=\frac{1}{\sqrt{1+\frac{v_{\chi^{\prime}}^{2}}{v_{\phi}^{2}}}}\left(I_{\phi}-\frac{v_{\chi^{\prime}}}{v_{\phi}} I_{\chi^{\prime}}\right), \\
& G_{1}=\sqrt{\frac{\xi}{v_{\phi}^{2} v_{\chi^{\prime}}^{2}+\xi}}\left(I_{\rho}-\frac{v_{\phi}^{2} v_{\chi^{\prime}}}{\xi} I_{\chi^{\prime}}-\frac{v_{\phi} v_{\chi^{\prime}}^{2}}{\xi} I_{\phi}\right), \\
& G_{2}=\frac{1}{\sqrt{1+\frac{v_{\chi^{\prime}}^{2}}{v_{\eta}^{2}}}}\left(I_{\eta^{\prime}}+\frac{v_{\chi^{\prime}}}{v_{\eta}} I_{\chi}\right), \\
& G_{3}=\sqrt{\frac{v_{\eta}^{2}\left(v_{\phi}^{2} v_{\chi^{\prime}}^{2}+\xi\right)}{v_{\rho}^{2} v_{\phi}^{2} v_{\chi^{\prime}}^{2}+v_{\eta}^{2}\left(v_{\phi}^{2} v_{\chi^{\prime}}^{2}+\xi\right)}}\left(I_{\eta}-\frac{v_{\rho}^{2} v_{\phi}^{2} v_{\chi^{\prime}}}{v_{\eta}\left(v_{\phi}^{2} v_{\chi^{\prime}}^{2}+\xi\right)} I_{\chi^{\prime}}\right. \\
& \left.-\frac{v_{\rho} v_{\phi}^{2} v_{\chi^{\prime}}^{2}}{v_{\eta}\left(v_{\phi}^{2} v_{\chi^{\prime}}^{2}+\xi\right)} I_{\rho}-\frac{v_{\rho}^{2} v_{\phi} v_{\chi^{\prime}}^{2}}{v_{\eta}\left(v_{\phi}^{2} v_{\chi^{\prime}}^{2}+\xi\right)} I_{\phi}\right) \\
& P S_{1}=\frac{1}{\sqrt{1+\frac{v_{\phi}^{2}}{v_{\eta}^{2}}+\frac{v_{\phi}^{2}}{v_{\rho}^{2}}+\frac{v_{\phi}^{2}}{v_{\chi^{\prime}}^{2}}}} \\
& \times\left(I_{\phi}+\frac{v_{\phi}}{v_{\chi^{\prime}}} I_{\chi^{\prime}}+\frac{v_{\phi}}{v_{\eta}} I_{\eta}+\frac{v_{\phi}}{v_{\rho}} I_{\rho}\right) \\
& P S_{2}=\frac{1}{\sqrt{1+\frac{v_{\eta}^{2}}{v_{\chi^{\prime}}^{2}}}}\left(I_{\eta^{\prime}}-\frac{v_{\eta}}{v_{\chi^{\prime}}} I_{\chi}\right),
\end{aligned}
$$


where we have defined $\xi \equiv v_{\rho}^{2}\left(v_{\phi}^{2}+v_{\chi^{\prime}}^{2}\right)$. In the above equation, the axion is identified as $a$, and the remaining three Goldstone bosons $G_{1}, G_{2}$, and $G_{3}$ together with the Goldstone boson in Eq. (24) are those absorbed by the four neutral gauge bosons of the model. The last two linear combinations of the interaction states in Eq. (27), $P S_{1}$ and $P S_{2}$, are the massive eigenstates or pseudo-Goldstone bosons. The important point that can be extracted and from these results is that our axion has a small component of $I_{\chi^{\prime}}$. Since, $v_{\phi}$ $\simeq 10^{10} \mathrm{GeV}$ and $v_{\chi^{\prime}} \simeq 10^{3} \mathrm{GeV}$, this component is very suppressed and, as expected, our axion is invisible, being almost exclusively the imaginary part of the singlet $\phi$. Besides, since $I_{\chi^{\prime}}$ couples only to the exotic quarks, our axion is very different even from that obtained in the version of 3-3-1 in Ref. [19], which does couple to neutrinos at the tree level. Its coupling can be easily obtained after rotating the mass eigenstates, Eq. (27), in terms of the interaction eigenstates, and it translates into the Lagrangian term

$$
\mathcal{L}_{a q^{\prime} q^{\prime}}=\frac{-i v_{\chi^{\prime}}}{\sqrt{2\left(v_{\phi}^{2}+v_{\chi^{\prime}}^{2}\right)}}\left[G_{1} \bar{u}_{3 L^{\prime}}^{\prime} u_{3 R}^{\prime}-G_{2}^{i j} \bar{d}_{i L}^{\prime} d_{j R}^{\prime}\right] a+\text { H.c. },
$$

which is very weak for $G_{1}, G_{2}^{i j} \sim$ of the order of unity, since $v_{\phi} \gg v_{\chi^{\prime}}$. The pseudo-Goldstone bosons $P S_{1}$ and $P S_{2}$ are more strongly coupled to fermions and, differently from the axion, also couple to ordinary matter. This leads us to conclude that, in this model, the only candidate for cold dark matter is the axion. We could check if the real massive scalars could fit for this role also, but a rough numerical approximation just confirms that they behave as their partners, as we could expect.

We also checked the coupling of our axion with photons. It is defined through the effective Lagrangian term

$$
\mathcal{L}_{a \gamma \gamma}=\frac{\bar{c}_{a \gamma \gamma}}{32 \pi^{2}} \frac{a(x)}{v_{P Q}} \widetilde{F}_{\mu \nu} F^{\mu \nu}
$$

In the present model only exotic quarks participate in the loop, leading to the above anomaly term, which leads to

$$
\bar{c}_{a \gamma \gamma}=-\frac{2}{3 v_{P Q}} \sum_{q^{\prime}} X_{q^{\prime}} Q_{q^{\prime}}^{2}=\frac{4}{9} \simeq 0.44 .
$$

This value is very similar to those obtained in different models present in the literature and can be used to make the relevant computations involving axions in astrophysical processes.

\section{SUMMARY AND CONCLUSIONS}

We studied the consequences of discrete symmetries in the version of the 3-3-1 model with right-handed neutrinos. One of the main points in this work is that global symmetries appear automatically as a consequence of such discrete symmetries in this model. It turned out that, when the model has a $Z_{11} \otimes Z_{2}$ symmetry, the whole Lagrangian is invariant under $U(1)$ transformations and also total lepton number is con- served at the classical level. It is remarkable that this happens in this more economical version of the model by adding only one singlet scalar; no other fields are necessary, which makes it a suitable model for implementing the strong- $C P$ problem solution.

We then recognized the global symmetry identifying it with a chiral PQ symmetry $U_{P Q}(1)$. In general, solutions to the strong- $C P$ problem through the PQ mechanism lead to domain wall formation, which is a threatening feature to cosmology, but fortunately model dependent. In this version of the 3-3-1 model this problem is absent due to the fact that we chose a relation among PQ charges-namely, $X_{d}=X_{d}^{\prime}$-which avoids this situation. Nevertheless, we have to remark that other possibilities would be allowed if we had not imposed a $Z_{2}$ symmetry. In this case, we could have let $X_{d}^{\prime}$ free and, working with the non-Hermitian potential terms, look for the relations among the PQ charges that would keep the $U_{P Q}(1)$ invariance. Such a relation exists and is given by $3 X_{d}^{\prime}=-X_{d}$, which is consistent with PQ invariance for all three $Z_{11}$ invariant non-Hermitian terms $\chi^{\dagger} \eta \phi^{*} \phi^{*}, \eta \rho \chi \phi$, and $\eta \eta \rho \phi^{*}$. In this situation we would have to address the formation of domain walls, and for this reason our previous choice seemed physically more appealing.

We proceeded with the spontaneous breaking of $U_{P Q}(1)$ and studied its consequences, obtaining the axion and showing that it is mainly constituted of the singlet. It was shown that it interacts with exotic quarks only, through a very suppressed coupling. Hence, our axion is an invisible one. However, since global symmetries are not stable against gravity effects, our axion could be in danger, receiving large mass corrections and losing its appeal as a CDM candidate. This was circumvented by assuming that $Z_{11}$ is a subgroup of an underlying gauge group at some high-energy scale. Annoying gravity-induced terms contributing to the axion mass are conveniently suppressed thanks to the local $Z_{11}$, stabilizing the axion. Although larger discrete symmetries would lead to a better stabilization of the axion against gravity, as well as a $\theta_{\text {eff }}$ safer from experimental constraints, we saw that it is still possible to have a $Z_{11}$ leading to small mass corrections and $\theta_{\text {eff }}$ below the bound $\theta_{\text {eff }}<10^{-9}$ if $v_{\phi} \equiv\langle\phi\rangle$ $\simeq 10^{10} \mathrm{GeV}$. Besides, the fact that 11 is a prime number allows $\phi$ to acquire any charge under $Z_{11}$ except the trivial one. This implies no need of assigning a very specific charge to $\phi$ in order to avoid restriction to smaller subsets of $Z_{N}$ which would not lead to axion stabilization.

Finally, there is a point that has to be highlighted when considering the 3-3-1 version with right-handed neutrinos. In the form it was presented here the model generates arbitrary masses for two neutrinos only, which can be deduced from the antisymmetry of the Yukawa coupling $h_{a b}^{\prime}$ in Eq. (5). This leads to an antisymmetric neutrino mass matrix, implying a zero eigenvalue and two degenerate ones. Although the massiveness of neutrinos is not the issue here, it would be nice to have a model that at least produces an appropriate arbitrary mass pattern for all fermions of the theory. To accomplish this we have to devise some way of eliminating such mass degeneracy. We can suggest two ways of doing that. One of them makes no enlargement of the spectrum and 
seems the preferable one, dealing only with the vacuum of the theory. In Ref. [20], the breaking of leptonic symmetry could be achieved only through a nonconserving PQ symmetry term-namely, the $\eta \rho \chi$ - when $\eta^{\prime}$ acquires a nonvanishing VEV. However, we have seen that our approach allows for an equivalent term which could lead to lepton number violation too, which is $\eta \rho \chi \phi$, with the difference that in this case PQ symmetry is still conserved. This term allows for a Majorana neutrino mass through loop corrections, making possible a mass matrix which is arbitrary enough to accommodate nondegenerate and nonzero neutrino masses. Another way out could be traced by including a singlet neutrino in the spectrum. Such a neutrino carries the exact quantum numbers to provide the required invariance under $Z_{11} \otimes Z_{2}$ and $U_{P Q}(1)$ and leave only nondegenerate massive neutrinos in the theory. This second possibility sounds appealing since not many fields can be introduced without jeopardizing the desired discrete and global symmetries here studied. Whatever nature's choice, both would be adequate to fit in our approach.
Summarizing, we obtained automatic $U_{P Q}(1)$ and lepton number symmetries by imposing a local discrete symmetry in an economic version of 3-3-1 model with right-handed neutrinos. We got an invisible axion stabilized under gravitational mass corrections, absent of the domain wall problem, solving the strong- $C P$ problem and constituting a strong candidate to CDM. These results are a remarkable achievement of our work, considering that the only additional ingredient we have used was the inclusion of a singlet scalar in the model.

\section{ACKNOWLEDGMENTS}

We would like to thank Vicente Pleitez for useful discussions and suggestions. This research was partially supported by Fundação de Amparo à Pesquisa do Estado de São Paulo (FAPESP) (AGD,PSRS) and by Conselho Nacional de Desenvolvimento Científico e Tecnológico (CNPq) (CASP).
[1] J. Kim, Phys. Rep. 150, 1 (1987); R.D. Peccei, Adv. Ser. Dir. High Energy Phys. 3, 503 (1989); J.E. Kim, Prog. Part. Nucl. Phys. 45, 157 (2000).

[2] G. 't Hooft, Phys. Rev. Lett. 37, 8 (1976); Phys. Rev. D 14, 3432 (1976).

[3] Particle Data Group, K. Hagiwara et al., Phys. Rev. D 66, 010001 (2002).

[4] R.D. Peccei and H.R. Quinn, Phys. Rev. Lett. 38, 1440 (1977); Phys. Rev. D 16, 1791 (1977).

[5] J. Preskill, M.B. Wise, and F. Wilczek, Phys. Lett. 120B, 127 (1983); L.F. Abbott and P. Sikivie, ibid. 120B, 133 (1983); M. Dine and W. Fischler, ibid. 120B, 137 (1983); G. Raffelt and D. Seckel, Phys. Rev. Lett. 60, 1793 (1988); M.S. Turner, ibid. 60, 1797 (1988); R. Mayle et al., Phys. Lett. B 203, 188 (1988).

[6] S. Weinberg, Phys. Rev. Lett. 40, 223 (1978); F. Wilczek, ibid. 40, 279 (1978).

[7] R.D. Peccei, in Proceedings of the 19th ICHEP Meeting, Tokyo, 1978, edited by H. Homma et al. (Physical Society of Japan, Tokyo, 1979), p. 1045.

[8] J.E. Kim, Phys. Rev. Lett. 43, 103 (1979); M.A. Shifman, A.I. Vainshtein, and V.I. Zakharov, Nucl. Phys. B166, 493 (1980).

[9] M. Dine, W. Fischler, and M. Srednicki, Phys. Lett. 104B, 199 (1981); A.P. Zhitnitskii, Sov. J. Nucl. Phys. 31, 260 (1980).

[10] M. Srednicki and N.J.C. Spooner, in [3]; A.D. Lewis, D.A. Buote, and J.T. Stocke, Astrophys. J. 586, 135 (2003), and references therein.

[11] F. Pisano and V. Pleitez, Phys. Rev. D 46, 410 (1992); P.H. Frampton, Phys. Rev. Lett. 69, 2889 (1992).

[12] R. Foot, H.N. Long, and T.A. Tran, Phys. Rev. D 50, R34 (1994); J.C. Montero, F. Pisano, and V. Pleitez, ibid. 47, 2918 (1993).

[13] L. Clavelli and T.C. Yang, Phys. Rev. D 10, 658 (1974); B.W. Lee and S. Weinberg, Phys. Rev. Lett. 38, 1237 (1977); B.W. Lee and R.E. Shrock, Phys. Rev. D 17, 2410 (1978); M.
Singer, ibid. 19, 296 (1979); M. Singer, J.W.F. Valle, and J. Schechter, ibid. 22, 738 (1980).

[14] R. Foot, O.F. Hernandez, F. Pisano, and V. Pleitez, Phys. Rev. D 47, 4158 (1993); D. Ng, ibid. 49, 4805 (1994); J.T. Liu, ibid. 50, 542 (1994); F. Pisano, J.A. Silva-Sobrinho, and M.D. Tonasse, Phys. Lett. B 388, 338 (1996); M.D. Tonasse, ibid. 381, 191 (1996); M. Ozer, Phys. Rev. D 54, 4561 (1996); P. Das, P. Jain, and D.W. McKay, ibid. 59, 055011 (1999); J.C. Montero, V. Pleitez, and O. Ravinez, ibid. 60, 076003 (1999); Y. Okamoto and M. Yasue, Phys. Lett. B 466, 267 (1999); G. TavaresVelasco and J.J. Toscano, ibid. 472, 105 (2000); P.H. Frampton and A. Rasin, ibid. 482, 129 (2000); G. Tavares-Velasco and J.J. Toscano, Europhys. Lett. 53, 465 (2001); M.B. Tully and G.C. Joshi, Phys. Rev. D 64, 011301 (2001); J.C. Montero, C.A. de S. Pires, and V. Pleitez, ibid. 66, 113003 (2002); D. Fregolente and M.D. Tonasse, Phys. Lett. B 555, 7 (2003); I. Gogoladze, Y. Mimura, and S. Nandi, ibid. 554, 81 (2003).

[15] H.N. Long, Phys. Rev. D 54, 4691 (1996); H. Ngoc Long and V.T. Van, J. Phys. G 25, 2319 (1999); N.A. Ky, H.N. Long, and D.V. Soa, Phys. Lett. B 486, 140 (2000); H.N. Long and D.V. Soa, Nucl. Phys. B601, 361 (2001); D.T. Binh, D.T. Huong, T.T. Huong, H.N. Long, and D.V. Soa, J. Phys. G 29, 1213 (2003).

[16] These new exotic quarks of both versions carry baryon and lepton numbers as well and are fairly recognized as leptoquarks.

[17] C.A. de S. Pires and O.P. Ravinez, Phys. Rev. D 58, 35008 (1998); C.A. de S. Pires, ibid. 60, 075013 (1999).

[18] P.B. Pal, Phys. Rev. D 52, 1659 (1995).

[19] Alex G. Dias, V. Pleitez, and M.D. Tonasse, Phys. Rev. D 67, 095008 (2003); Alex G. Dias and V. Pleitez, hep-ph/0308037.

[20] C.A. de S. Pires and P.S. Rodrigues da Silva, hep-ph/0307253.

[21] H.N. Long and N.Q. Lan, Europhys. Lett. 64, 571 (2003).

[22] L.M. Krauss and F. Wilczek, Phys. Rev. Lett. 62, 1221 (1989).

[23] M. Kamionkowski and J. March-Russell, Phys. Lett. B 282, 137 (1992). 
[24] M. Dine, in "Proceedings of the Cincinnati Symposium on Quantum Gravity and Beyond," hep-ph/9207045, pp. 157169; T. Banks, M. Dine, and M. Graesser, Phys. Rev. D 68, 075011 (2003).

[25] C.T. Hill and A.K. Leibovich, Phys. Rev. D 66, 016006 (2002); 66, 075010 (2002).

[26] E.J. Chun and A. Lukas, Phys. Lett. B 297, 298 (1992).

[27] Alex G. Dias, V. Pleitez, and M.D. Tonasse, hep-ph/0210172.

[28] Observe that $\chi$ and $\eta$ transforming in the same way should have similar couplings with matter. However, the interactions here presented are those which generate the desired mass pattern for the fermions. Later we will see that they are the only ones that will survive under the discrete symmetries of the model.
[29] We are omitting the neutral current interactions here because throughout this work we will be dealing only with interactions which might provide some global quantum number violation.

[30] G. Lazarides, C. Panagiotakopoulos, and Q. Shafi, Phys. Rev. Lett. 56, 432 (1986).

[31] R.D. Peccei, Nucl. Phys. B (Proc. Suppl.) 72, 3 (1999); Proceedings of 4th International Symposium on Sources and Detection of Dark Matter in the Universe (DM 2000), Marina del Rey, California, 23-25 February 2000, in "Marina del Rey 2000 , Sources and detection of dark matter and dark energy in the universe," pp. 98-104, hep-ph/0009030.

[32] Y.B. Zeldovich, I.B. Kobzarev, and L.B. Okun, Sov. Phys. JETP 40, 1 (1975); Phys. Lett. 50B, 340 (1974); P. Sikivie, Phys. Rev. Lett. 48, 1156 (1982); H. Georgi and M.B. Wise, Phys. Lett. 116B, 123 (1982). 